\title{
Spatial pattern learning in the radial arm maze
}

\author{
MICHAEL F. BROWN and GARY W. GIUMETTI \\ Villanova University, Villanova, Pennsylvania
}

\begin{abstract}
Rats experienced a spatial pattern of baited and unbaited arms in an eight-arm radial maze. The spatial pattern remained constant over trials, but the spatial locations that were baited varied unpredictably. Although there was no evidence of control by the spatial pattern during free choice training trials, the rats' ability to locate baited arms in forced choice test trials was superior to that of animals in a control condition for which maze arms were not baited in a consistent spatial pattern. This is consistent with the results of experiments showing that spatial choices by rats in a pole box maze are controlled by abstract spatial patterns.
\end{abstract}

A series of experiments from our laboratory has demonstrated that spatial choices in rats can be controlled by the spatial relations among hidden goal locations (Brown, DiGello, Milewski, Wilson, \& Kozak, 2000; Brown \& Terrinoni, 1996; Brown, Zeiler, \& John, 2001). These experiments involve a spatial task known as the pole box maze, in which rats search for food pellets hidden on top of vertical poles arranged in a two-dimensional matrix. An important aspect of this work is that the spatial relations controlling behavior are not among particular places and are therefore not defined by, or anchored to, the visual or other perceptual cues that correspond to particular places. Because the locations baited on individual trials vary unpredictably over trials, the learned spatial relations must be abstracted from spatial relations among particular places, possibly by means of a path integration process (Brown, 2006b). On individual trials, working memory for bait status of previously visited locations in combination with the learned spatial pattern provides information about the location of baited locations that remain (Brown, 2006b; DiGello, Brown, \& Affuso, 2002).

Dallal and Meck (1990; Experiment 3) reported evidence of similar control of spatial choices by abstract spatial relations in the radial arm maze. Dallal and Meck trained rats in a 12 -arm maze. For each rat, 8 arms were baited and 4 were not. The bait status of a maze arm remained constant during training, but the identity of baited and unbaited arms varied over subjects. Thus, it was possible for the rats to learn the spatial pattern of baited and unbaited arms. Evidence that they did so was obtained by testing the rats in the same maze placed in a second extra-maze environment. The rats in one of the two relevant experimental groups had the same spatial arrangement of baited and unbaited arms in the transfer test that

This project was supported by National Science Foundation Grant BNS-9982244. The authors thank Urmo Jaanimägi, who conducted pilot research critical for the development of this project. Correspondence should be addressed to M. F. Brown, Department of Psychology, Villanova University, Villanova, PA 19085 (e-mail: michael.brown@ villanova.edu). they had had during training. Rats in a second group had a different spatial arrangement of baited and unbaited arms. Although neither group could use spatial cues defining specific maze arms in the transfer test, it was possible for the former group to use the spatial relations among baited arms (hypothetically learned during training) to locate baited arms in the transfer test. The finding that transfer performance was superior in that group was interpreted by Dallal and Meck as evidence that they had done so. Like our results obtained with the pole box maze, this finding indicates that the spatial representations controlling choices are independent of any particular spatial locations and are therefore properly considered to be of the spatial relations themselves (in the abstract) rather than of spatial relations anchored to particular spatial locations.

Olthof, Sutton, Slumskie, D'Addetta, and Roberts (1999) reported a systematic attempt to replicate and extend Dallal and Meck's (1990) finding. In two experiments, they failed to find evidence for the basic effect reported by Dallal and Meck. In three additional experiments, Olthof et al. looked for transfer of control by a spatial pattern by training rats in a 12 -arm radial maze with one configuration of baited and unbaited arms and then evaluating performance when the configuration was rotated so that, although the configuration remained the same, the baited and unbaited arms corresponded to different spatial locations in the extra-maze environment. These experiments also provided no evidence for control by a spatial pattern. Olthof et al. concluded that "there is little evidence to support the argument that rats learn overall geometric patterns" (p. 361).

A possible limitation of the approach taken by Dallal and Meck (1990, Experiment 3) and of the experiments reported by Olthof et al. (1999) is that the rats were trained in a procedure in which specific locations (defined by specific perceptual cues), as well as the spatial pattern of baited and unbaited arms, predicted the location of baited arms. Because both kinds of information were relevant, it may be that they competed for behavioral control. If so, control by the spatial pattern in the transfer tests was not found because of the competition for behavioral control 
by spatial cues during training. A more appropriate strategy for investigating the possibility of control of choices in the radial maze by spatial patterns might be to arrange for the spatial pattern to be the only cue relevant to the location of baited arms that can be learned over trials, by rendering the location of baited arms on any particular trial irrelevant. This is essentially the strategy used in the pole box maze experiments (Brown \& Terrinoni, 1996). In those experiments, the baited locations varied unpredictably from trial to trial and only the spatial relations among the baited locations remained constant over trials.

In the present experiment, rats were trained in an eightarm radial maze in which four arms were baited and the remaining four were unbaited at the beginning of each trial. For one group of rats, the baited and unbaited arms always formed an alternating pattern; baited arms were separated from each other by one unbaited arm. Thus, there were two exemplars of this pattern. The exemplar used on any particular trial was selected randomly. As a result, each of the eight locations was baited on half of the trials and was therefore not predictive of the bait status of a maze arm. The alternating spatial pattern of baited and unbaited arms, however, was constant across trials. A second group of rats was trained in a no-pattern control condition. A randomly selected set of four maze arms was baited on each trial. Thus, in the no-pattern condition, neither spatial locations nor spatial patterns were informative about which maze arms were baited.

After training under these conditions, the ability of these two groups of rats to locate baited arms was assessed in forced choice tests. In each of these test trials, the rat first visited two baited maze arms, which were randomly chosen from among the four baited arms. After a brief interruption of the trial, during which the rat was removed from the maze, it then chose from among the eight maze arms until it located the two remaining baited arms. Under these conditions, the ability of rats in the no-pattern condition to locate the two remaining baited arms (among the six arms that had not been visited prior to the trial's interruption) should have been no better than expected on the basis of chance. However, if rats in the alternation pattern condition had learned the pattern, then the learned pattern in combination with memory for the location of the two baited arms visited before removal from the maze should provide information about the location of the remaining baited arms.

\section{METHOD}

\section{Subjects}

The subjects were 12 male Sprague Dawley rats, obtained from Harlan Sprague Dawley (Indianapolis) as weanlings. They had been housed in pairs and provided with free access to water and food until they were 12 weeks old. Experimental trials occurred during the dark phase of a 12:12-h light:dark cycle.

\footnotetext{
Apparatus

The apparatus was a radial maze with eight arms. The central arena, $25 \mathrm{~cm}$ tall and $46 \mathrm{~cm}$ in diameter, was constructed of plastic and had a floor that consisted of a thick layer of the same wood chips
}

that were used in the rats' home cages. Eight equally spaced circular holes $(10.0 \mathrm{~cm}$ in diameter, centered $6.5 \mathrm{~cm}$ above the floor) led to the arms, which were tubes constructed of white PVC $10 \mathrm{~cm}$ in diameter and $90 \mathrm{~cm}$ long. The terminal $10 \mathrm{~cm}$ of each tube was cut so that only half of the circumference of the tube was present (the bottom half). This created a platform on the end of each arm, from which visual cues were fully available and on which a small $(4.5-\mathrm{cm}$-square and 0.9-cm-deep) translucent plastic dish was mounted as a food cup. It is important to emphasize that, although the maze arms were enclosed, access to visual extra-maze cues was available from both the central arena and the platforms at the end of each maze arm. This differs from similarly constructed apparatus that have been used in earlier experiments in our laboratory (Brown \& Moore, 1997). The construction of the apparatus used in the present experiment was intended to allow the subjects to use spatial working memory to code the locations visited during trials, just as rats are believed to do in most radial arm apparatus.

Maze arms were baited by placing two 45-mg sucrose pellets (Noyes, Product PJFSC000045) in the food cup. Each maze arm could be made inaccessible by placing a stopper in the arm (in the end of the arm attached to the central arena). The stopper consisted of a white plastic flower pot inserted bottom-end-first so that the bottom formed a wall $9.5 \mathrm{~cm}$ into the maze arm.

The maze was centered in a $36.1 \times 35.3 \mathrm{~m}$ room with a variety of objects around the perimeter and illuminated by fluorescent tubes. A video camera mounted above the central arena of the maze allowed the experimenter to monitor the behavior of the rats in the central arena from his station in one corner of the room.

\section{Preliminary Training Procedure}

The rats were placed on a restricted diet and their weights were monitored throughout the experiment so that they could be maintained at $85 \%-95 \%$ of free-feeding weight. Beginning 7 days before being first placed in the maze, the rats were given several sucrose pellets in their home cages daily. One rat from each pair of cage mates was randomly assigned to the alternation pattern condition, and the other was assigned to the no-pattern control condition.

For four daily sessions, pairs of rats (cagemates) were placed in the maze for $10 \mathrm{~min}$. At the beginning of each session, there were two pellets in each food cup and approximately eight pellets in the central arena.

\section{Free Choice Training Phase}

Prior to each trial, two pellets were placed in the food cup at the end of each of four baited maze arms. For the rats in the no-pattern control condition, the four baited arms were randomly selected for each trial. For the rats in the alternation pattern condition, one of the two pattern exemplars (the two possible configurations of four arms, all separated by one unbaited arm) was randomly selected for each trial and the corresponding four maze arms were baited. The rat was then placed in the central arena of the maze and allowed to choose maze arms until all four baited arms had been chosen or $10 \mathrm{~min}$ had elapsed. A choice was defined when all four paws were on the surface of the maze arm. The experimenter recorded the sequence of arms chosen. For each rat, one trial per day was conducted for 20 days.

\section{Forced Choice Test Phase}

Prior to each trial, four baited arms were selected as baited using the same procedure as in the free choice training trials. Two of the baited arms were randomly selected to be available during the forced choice phase of the trial. The remaining six maze arms were made inaccessible using the stoppers described above. The rat was placed in the central arena until both accessible arms were chosen or until $4 \mathrm{~min}$ had elapsed. The rat was then removed from the central arena of the maze and placed in a holding cage while the experimenter removed the plugs from the maze arms. The rat was then returned 
to the maze. The interval from removal of the rat from the maze to its return to the central arena was approximately $1 \mathrm{~min}$. The rat was then allowed to choose arms until both remaining baited arms were chosen or until $4 \mathrm{~min}$ had elapsed. The experimenter recorded the sequence of arms chosen. One trial per day was conducted for 15 days.

\section{RESULTS}

\section{Free Choice Training Phase}

One subject in the no-pattern control group replaced a rat that failed to consistently obtain pellets from the ends of maze arms. The replacement subject received only 13 trials of free choice training. Its data are not included in the analyses of the free choice training results, but are included in the analysis of test data. The four baited maze arms were chosen within the trial time-out limit during all but 4 trials (out of 220).

To determine whether baited arms were chosen earlier in the choice sequence than unbaited arms, the mean serial position of the initial choice to each of the eight maze arms was determined for each trial. For cases in which all eight arms were not chosen during a trial (either because the rat did not choose all four baited maze arms within the trial time-out limit or because some unbaited arms had not been chosen when the rat completed the trial), unchosen $\operatorname{arm}(\mathrm{s})$ were assigned a serial position value that was the mean of the remaining choice serial positions. Thus, the mean serial position of the eight maze arms was (necessarily) 4.5 for each trial. Figure 1 shows the mean serial position of choices to baited arms over the course of four trial blocks for the two experimental groups. A trial block $\times$ group ANOVA provided no evidence for an effect of block $[F(3,27)<1]$ or group $[F(1,9)<1]$, or an interaction between the effects of these variables

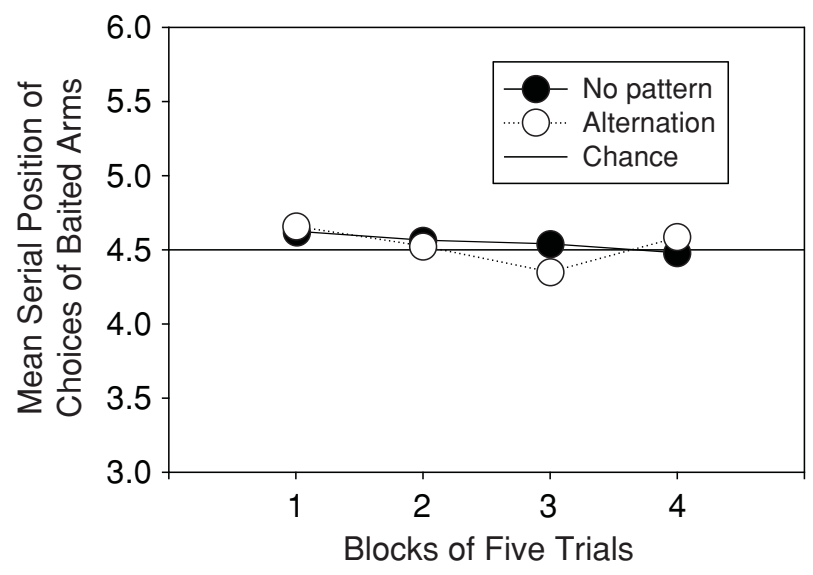

Figure 1. The mean serial position of the first visit made to baited arms during the free choice training phase for the two experimental groups. For each trial, each maze arm was assigned a serial position value that was either the serial position of the first visit to that arm or (if the arm did not receive a visit during the trial) the mean of the remaining serial positions. Thus, the mean serial position for all maze arms (and the value expected on the basis of chance) is 4.5 .
$[F(3,27)<1]$. The mean serial position of choices to baited arms (collapsed over groups and trial blocks = 4.46) does not differ from the value expected on the basis of chance $(4.5)[t(11)<1]$.

To determine whether rats in the alternation pattern group acquired a tendency to choose arms separated from the last maze arm chosen by one other maze arm (i.e., a spatial alternation pattern of choices), the distributions of spatial separations between consecutive choices were determined. For each choice that was preceded by the initial choice of another maze arm, the spatial separation between that choice and the preceding choice was determined (cases in which the preceding choice was a revisit to a maze arm chosen earlier in the trial were excluded from the analysis because of the psychologically ambiguous bait status of that arm). Figure 2 shows the proportions of choices made to maze arms that were adjacent to (separation $=1$ ) and separated by one maze arm from (separation $=2$ ) the most recently chosen maze arm. The proportions are shown as a function of experimental group and the bait status of the previously chosen maze arm. Response tendencies that conform to the alternating pattern of baited arms would be indicated by relatively greater tendencies to choose a spatially adjacent maze arm following choice of an unbaited arm and to choose a maze arm separated by one arm from the preceding choice following choice of a baited arm. An ANOVA comparing the proportions of choices as a function of experimental group, spatial separation, bait status of preceding choice, and trial block found no evidence for effects of any of these variables or for any interactions among them [block, $F(3,27)=2.88$; block $\times$ group, $F(3,27)=1.75$; block $\times$ bait status, $F(3,27)=1.37$; all other $F \mathrm{~s}<1]$.

\section{Forced Choice Test Phase}

The two baited maze arms available prior to the trial interruption were chosen during every trial. The two remaining baited arms were chosen following trial interruption within the trial time-out limit on all but five trials.

Following the forced choices and trial interruption, the rat chose from among the two (now unbaited) maze arms to which it had been forced prior to the trial interruption, the two baited arms, and the four unbaited arms. The relative tendency to choose each of these three categories of alternatives was assessed by determining the mean serial position of maze arms in each of the three categories. For cases in which all eight arms were not chosen following the trial interruption (either because the rat did not choose both baited maze arms within the allotted time or because some unbaited arms had not been chosen when the rat completed the trial), unchosen arms were assigned a serial position value that was the mean of the remaining choice serial positions.

Figure 3 shows the mean serial position of maze arms in the three categories for the two experimental groups. Because these values are not independent (the overall mean for each group is necessarily 4.5), the analysis focused on the two categories of maze arms not visited prior to the trial interruption. An ANOVA was used to compare the 


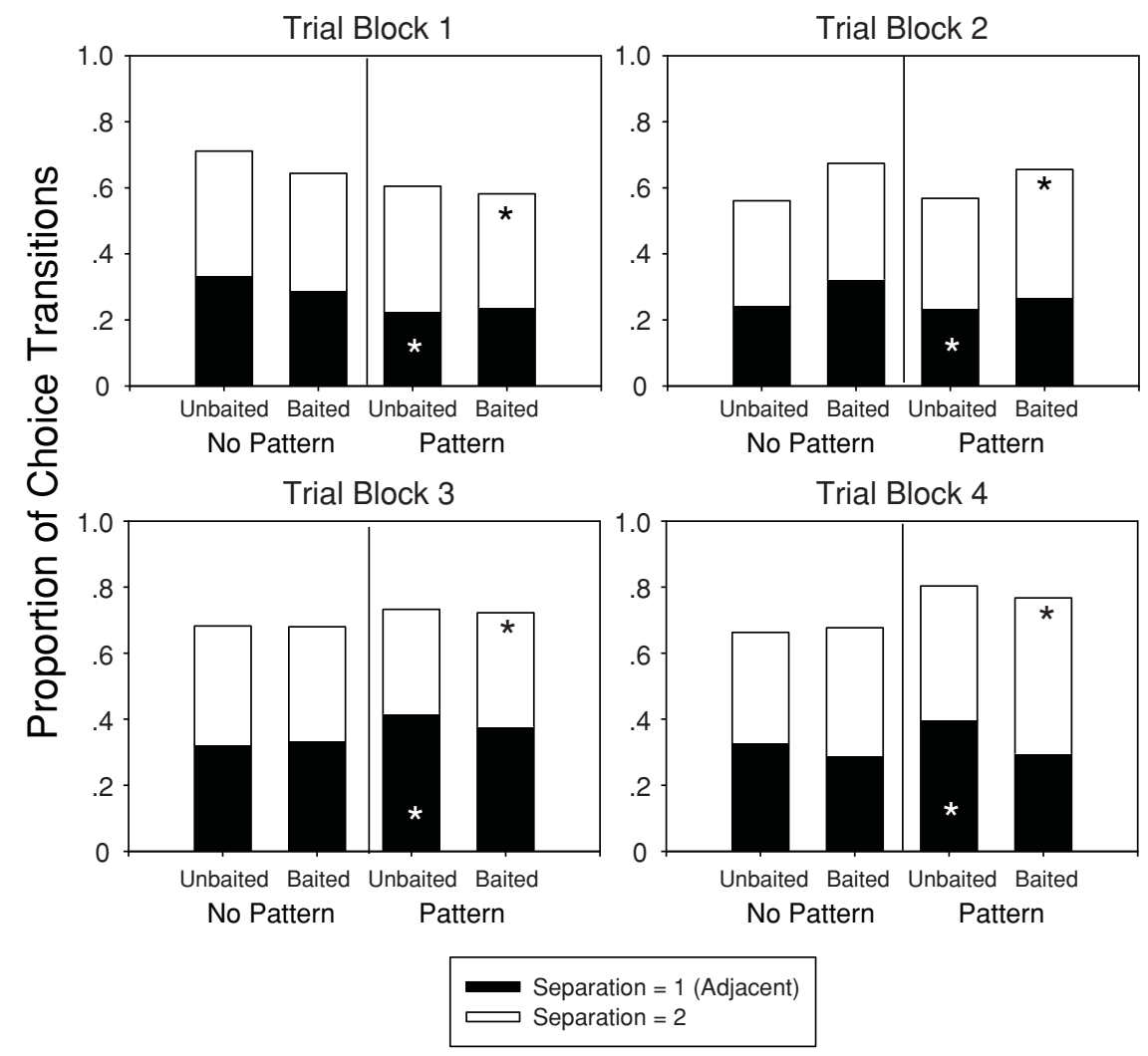

Figure 2. The spatial relations between consecutive choices during the four trial blocks of the free choice phase. Each bar shows the proportion of choices to maze arms adjacent to the maze arm last chosen (Separation $=1$ ) and the proportion separated from the last choice by one maze arm (Separation $=2$ ). These mean values are shown as a function of experimental group and the bait status of the maze arm last chosen. Asterisks mark the spatial relations between consecutive choices consistent with the spatial pattern.

mean serial positions of the two baited arms and the four unbaited arms in the two experimental groups. There was a significant group $\times$ bait status interaction $[F(1,10)=$ 9.4]. In the no-pattern group, the mean serial positions of baited and unbaited arms did not differ significantly $[t(5)=1.57]$. In the alternation pattern group, there was a significant tendency to visit the baited arms $[t(5)=4.72$, $p<.01]$.

In addition, the two arms visited by the rats during the forced choice phase of each trial were visited later in the choice sequence, if at all. Their mean serial position values (shown in Figure 3) were greater than expected on the basis of chance for both the alternation pattern group $[t(5)=5.8]$ and the no-pattern group $[t(5)=18.3]$.

A separate analysis considered the first choice of a previously unvisited arm following the trial interruption. The probability of choosing a baited arm by chance immediately following the trial interruption was .333 (two out of the six previously unvisited arms were baited). Rats in the alternation pattern group and no-pattern control group chose a baited arm on a mean (over rats) of .433 and .289 of trials, respectively. These values differ significantly $[t(10)=2.29, p<.05]$. The former value differs signifi- cantly from chance $[t(10)=3.5, p<.05]$ and the latter does not $[t(10)<1]$.

\section{DISCUSSION}

During the free choice training trials, there was no evidence that choices by rats in the alternation pattern condition were affected by the spatial pattern. That is, the rats did not acquire a tendency to visit the baited arms earlier in the choice sequence than expected by chance or earlier in the choice sequence than baited arms were chosen by rats in the no-pattern control group. Likewise, the spatial relation between arms chosen consecutively did not come to conform to the pattern. Control by the pattern would be expected to result in an increase in the tendencies to choose spatially adjacent arms following choice of an unbaited arm and arms separated by one other arm following choice of a baited arm. These tendencies did not develop.

However, during the forced choice test phase that followed free choice training, there was clear evidence that the choices of rats in the alternation pattern group were controlled by the pattern. After visiting two randomly chosen baited arms, rats in the alternation pattern chose the 


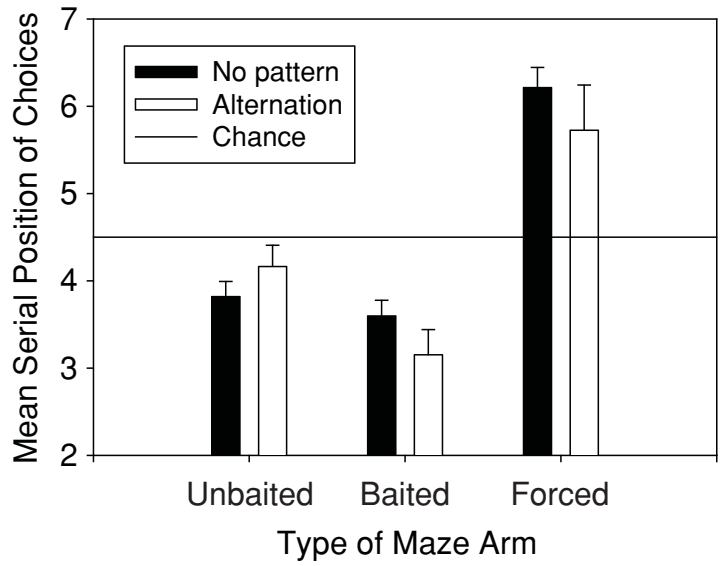

Figure 3. The mean serial position of the first visit made to baited arms following the trial interruption during the forced choice test phase for the two experimental groups. For each trial, each maze arm was assigned a serial position value that was either the serial position of the first visit to that arm after the rat was returned to the maze or (if the arm did not receive a visit following the trial interruption) the mean of the remaining serial positions. Thus, the mean serial position for all maze arms (and the value expected on the basis of chance) is 4.5. Error bars show 1.0 standard error of the mean.

two remaining baited arms (which conformed to the spatial pattern) earlier in the choice sequence than they chose the unbaited arms (which did not conform to the pattern). This performance can be explained if the rats acquired a representation of the spatial pattern of alternating baited and unbaited arms. This pattern, in combination with memory for location(s) of one or both of the baited maze arms visited during the forced choices, allowed the rats to locate the two remaining baited arms more efficiently than rats in the no-pattern control condition.

An alternative to representation of the spatial alternation pattern is the possibility that rats in the pattern condition acquired a simple response tendency - move to an arm separated by one from the last choice following choice of a baited arm. The role of such response tendencies is a longstanding issue in the interpretation of performance in the radial arm maze (see, e.g., Olton, 1978). However, the tendency to choose maze arms conforming to the spatial alternation pattern in the present experiment cannot be accounted for by the development of a tendency to move from one maze arm to another in a consistent manner. Three considerations argue against this possibility. First, the forced choice aspect of the testing procedure was designed to disrupt any response tendencies that might have developed during free choice training. Second, there was no evidence that any response tendencies developed during training differed in the two experimental groups. Third, and most critically, the first choice made following the trial interruption was under the control of the alternation pattern, and it is not possible that the first choice made after the rat was removed from the maze, placed in the holding cage, and then returned to the maze could have been under the control of a response tendency.
The present data provide clear evidence that the spatial pattern of alternating baited arms influenced choices during the forced choice test phase of the experiment. It is somewhat puzzling that there was no indication of the acquisition of that control during the free choice training phase of the experiment. It may simply be that the amount of experience with the pattern necessary for it to control choices roughly corresponds to the duration of the training phase we used and that control would have been revealed in additional free choice training trials. Alternatively, there may be some feature of the forced choice testing procedure that elicits expression of control by the spatial pattern. One class of possibilities along these lines involves the application of different choice criteria during free choice and forced choice trials in the radial maze. It has been shown that choice accuracy in the radial-arm maze depends on the amount of time or effort required to visit a maze arm, with greater difficulty resulting in increased accuracy (Brown \& Huggins, 1993; Brown \& Lesniak-Karpiak, 1993). These effects are best interpreted in terms of differences in a choice criterion. The information available that allows rats to discriminate correct from incorrect maze locations is used to a greater or lesser extent depending on the cost of making a choice. It is also well known that rats often choose maze arms in the radial arm according to stereotypic response patterns (e.g., consistently choosing adjacent maze arms) in the free choice procedure but cease doing so when a forced choice procedure is implemented and the stereotypic response pattern no longer results in high levels of accuracy (see, e.g., Dale, 1986; Foreman, 1985). These two phenomena, considered together, suggest that psychological processes may control choices under the more challenging forced choice procedure that are not effective under the free choice procedure. The use of a learned spatial pattern of baited locations might be one such process.

Comparison of the results of the present experiment with those reported by Olthof et al. (1999) supports the idea that spatial pattern learning did not occur in their experiments because of the predictive information provided by spatial cues corresponding to particular locations during training. Control by the spatial configurations of baited and unbaited arms in their experiments may have been overshadowed by the valid spatial cues that were also available. Thus, a critical aspect of the present experimental procedure may have been the fact that spatial cues were never available as predictors of the arms baited and unbaited at the beginning of the trial. Additional work will be required in order to determine whether this feature of the present experimental design was, in fact, critical. However, two implications of this possibility should be noted. First, there have been several recent suggestions that cue competition does not occur in spatial learning, at least not as it is understood to occur in other contexts (see, e.g., Pearce, Ward-Robinson, Good, Fussell, \& Aydin, 2001). Of particular note is that Brown, Yang, and DiGian (2002) reported a lack of overshadowing of spatial pattern learning by visual cues in the pole box maze task. Thus, cue competition between spatial patterns and visual spatial 
cues in the radial arm maze would be of theoretical interest. Second, although visual spatial cues did not distinguish the maze arms baited at the beginning of each trial from those not baited in the present experiment, the visual cues corresponding to maze arm locations were valid cues for arms previously visited within a trial and were presumably used by the rats to avoid revisits of arms within a trial as well as to code the locations of baited and/or unbaited locations visited earlier in the trial. The possibility that such cues were used in avoiding revisits but were not involved in coding the spatial relationships among baited arms is consistent with recent data from the pole box maze which provide evidence for such a dissociation in the use of visual cues (Brown \& Wintersteen, 2004).

We interpret the present results as revealing a spatial pattern learning ability in the radial maze task that is parallel to that used in the pole box maze. It should be noted, however, that there is a viable alternative interpretation of the present results. With only two exemplars of the spatial pattern used in the present experiment, it is possible that the rats learned to group the two sets of four maze arms into two categories or equivalence sets (Vaughan, 1988). If so, then determination of the bait status of one member (maze arm) of the category or equivalence set (during the first choice of a particular trial) could allow the rat to selectively visit other members of the set baited on that trial. This interpretation was suggested by Wathen and Roberts (1994) as an explanation for the ability of rats in their experiment to learn multiple sets of baited and unbaited maze arms that varied systematically over daily sessions of multiple trials (see also Cohen \& Bussey, 2003; Tremblay \& Cohen, 2005). Brown (2006b) argued that an interpretation of performance in the pole box maze in terms of equivalence set learning was a logical possibility, but feasible only in the case of spatial patterns with a small number of exemplars (such as the checkerboard spatial pattern used by Brown et al., 2001). Given that equivalence set learning does not provide a general explanation for the control by spatial patterns that occurs in the pole box maze and the similarity of the results from the pole box maze and those we report here, we conclude that spatial pattern learning is the best interpretation of the present results. However, it will require additional empirical work to determine the relationship between spatial pattern learning and equivalence sets of spatial locations.

Starting with the influential experiment of Suzuki, Augerinos, and Black (1980), a number of experiments have provided evidence that a spatial configuration of particular locations, defined by visual extra- or intramaze cues, can acquire behavioral control (e.g., Brown, Rish, VonCulin, \& Edberg, 1993; Cohen \& Bussey, 2003; Tremblay $\&$ Cohen, 2005; Vollmer-Conna \& Lemon, 1998). Representation of spatial relations among specific locations has been considered to be a defining property of a cognitive map (O’Keefe \& Nadel, 1978; Poucet, 1993; ThinusBlanc, 1996). The spatial relations controlling choices in the present experiment, like those involved in the control of spatial choices in the pole box maze, however, differ from representations of the spatial relationships among specific locations. They are independent of any particular set of locations and therefore cannot be anchored to specific locations. In this sense they are appropriately identified as abstract representations of spatial relations (Brown, 2006b; Olthof et al., 1999) and may have psychological processes in common with other forms of abstract representation in animals, including those corresponding to serial and temporal patterns (Brown, 2006a).

\section{REFERENCES}

Brown, M. F. (2006a). Abstracting spatial locations among global locations. In M. F. Brown \& R. G. Cook (Eds.), Animal spatial cognition: Comparative, neural, and computational approaches [Online]. Available at www.pigeon.psy.tufts.edu/asc/.

Brown, M. F. (2006b). Spatial patterns: Behavioral control and cognitive representation. In E. A.Wasserman \& T. R. Zentall (Eds.), Comparative cognition: A natural science approach to the study of animal intelligence (pp. 425-438). Oxford: Oxford University Press.

Brown, M. F., DiGello, E., Milewski, M., Wilson, M., \& Kozak, M. (2000). Spatial pattern learning in rats: Conditional control by two patterns. Animal Learning \& Behavior, 28, 278-287.

Brown, M. F., \& Huggins, C. K. (1993). Maze-arm length affects a choice criterion in the radial-arm maze. Animal Learning \& Behavior, 21, 68-72.

Brown, M. F., \& LeSNIAK-KARPIAK, K. B. (1993). Choice criterion effects in the radial-arm maze: Maze-arm incline and brightness. Learning \& Motivation, 24, 23-39.

Brown, M. F., \& Moore, J. A. (1997). In the dark II: Spatial choice when access to extrinsic spatial cues is eliminated. Animal Learning \& Behavior, 25, 335-346.

Brown, M. F., Rish, P. A., VonCulin, J. E., \& Edberg, J. A. (1993). Spatial guidance of choice behavior in the radial-arm maze. Journal of Experimental Psychology: Animal Behavior Processes, 19, 195-214.

Brown, M. F., \& Terrinoni, M. (1996). Control of choice by the spatial configuration of goals. Journal of Experimental Psychology: Animal Behavior Processes, 22, 438-446.

Brown, M. F., \& Wintersteen, J. (2004). Spatial patterns and memory for locations. Learning \& Behavior, 32, 391-400.

Brown, M. F., Yang, S. Y., \& DiGian, K. A. (2002). No evidence for overshadowing or facilitation of spatial pattern learning by visual cues. Animal Learning \& Behavior, 30, 363-375.

Brown, M. F., ZeILER, C., \& John, A. (2001). Spatial pattern learning in rats: Control by an iterative pattern. Journal of Experimental Psychology: Animal Behavior Processes, 27, 407-416.

Cohen, J., \& Bussey, K. (2003). Rats form cognitive maps from spatial configurations of proximal arm cues in an enclosed 4-arm radial maze. Learning \& Motivation, 34, 168-184.

Dale, R. H. I. (1986). Spatial and temporal response patterns on the eight-arm radial maze. Physiology \& Behavior, 36, 787-790.

Dallal, N. L., \& MEcK, W. H. (1990). Hierarchical structures: Chunking by food type facilitates spatial memory. Journal of Experimental Psychology: Animal Behavior Processes, 16, 69-84.

DiGello, E., Brown, M. F., \& Affuso, J. (2002). Negative information: Both presence and absence of spatial pattern elements guide rats' spatial choices. Psychonomic Bulletin \& Review, 9, 706-713.

ForEMAN, N. (1985). Algorithmic responding on the radial maze in rats does not always imply absence of spatial encoding. Quarterly Journal of Experimental Psychology, 37B, 333-358.

O'Keefe, J., \& NADEL, L. (1978). The hippocampus as a cognitive map. Oxford: Oxford University Press.

Olthof, A., Sutton, J. E., Slumskie, S. V., D’Addetta, J., \& RobERTS, W. A. (1999). In search of the cognitive map: Can rats learn an abstract pattern of rewarded arms on the radial maze? Journal of Experimental Psychology: Animal Behavior Processes, 25, 352-362.

Olton, D. S. (1978). Characteristics of spatial memory. In S. H. Hulse, H. Fowler, \& W. K. Honig (Eds.), Cognitive processes in animal behavior (pp. 341-374). Hillsdale, NJ: Erlbaum.

Pearce, J. M., Ward-Robinson, J., Good, M., Fussell, C., \& Aydin, A. (2001). Influence of a beacon on spatial learning based on the shape 
of the test environment. Journal of Experimental Psychology: Animal Behavior Processes, 27, 329-344.

Poucet, B. (1993). Spatial cognitive maps in animals: New hypotheses on their structure and neural mechanisms. Psychological Review, 100, 163-182.

Suzuki, S., Augerinos, G., \& Black, A. H. (1980). Stimulus control of spatial behavior on the eight-arm maze in rats. Learning \& Motivation, 11, 1-18.

Thinus-Blanc, C. (1996). Animal spatial cognition: Behavioural and brain approach. Singapore: World Scientific.

Tremblay, J., \& Cohen, J. (2005). Spatial configuration and list learning of proximally cued arms by rats in the enclosed four-arm radial maze. Learning \& Behavior, 33, 78-89.
VAUGHAN, W. (1988). Formation of equivalence sets in pigeons. Journal of Experimental Psychology: Animal Behavior Processes, 14, 36-42.

Vollmer-Conna, U. S., \& Lemon, J. (1998). Spatial configuration and proximal cues. Learning \& Motivation, 29, 102-111.

Wathen, C. N., \& Roberts, W. A. (1994). Multiple-pattern learning by rats on an eight-arm radial maze. Animal Learning \& Behavior, 22, 155-164.

(Manuscript received June 28, 2005;

revision accepted for publication September 2, 2005.) 